\title{
Lawn mower injuries as a cause of serious visual acuity impairment - Case reports
}

\author{
Monika Jasielska', Mateusz Winiarczyk', Paweł Bieliński', Jerzy Mackiewicz' \\ ${ }^{1}$ Department of Vitreoretinal Surgery, Medical University, Lublin, Poland
}

Jasielska M, Winiarczyk M, Bieliński P, Mackiewicz J, Lawn mower injuries as a cause of serious visual acuity impairment - Case reports. Ann Agric Environ Med. 2017; 24(2): 261-264. doi: 10.5604/12321966.1233569

\begin{abstract}
Objective. The aim of the study is to present four cases of lawn mowers injuries as a cause of serious visual acuity impairment. Materials and method. A retrospective study of four patients admitted in 2013-2015 to the Department of Vitreoretinal Surgery in Lublin with severe open or closed globe injury, one with an intraocular foreign body (IOFB). The presence of eye protective equipment was assessed, as well as visual acuity, eye tissue condition before and after treatment, and applied therapy.

Results. In all cases an improvement was achieved in local conditions. The intraocular foreign body was removed, wounds sutured and damaged tissues placed in position. All eyeballs were saved. In three cases, visual acuity was improved to a usable level. Three patients underwent pars plana vitrectomy, one with IOFB removal from the vitreous cavity.

Conclusions. Lawn mower induced eye injuries are a significant cause of serious visual acuity impairment or blindness. The presented study shows that lawn mower eye injuries are still a therapeutic, social and economic problem, yet are very preventable with proper eye protection and patients' education. Current prevention strategies are inadequate, and therefore should be updated.
\end{abstract}

\section{Key words}

ocular trauma, perforating eye injury, lawn mowers injury, intraocular foreign body, pars plana vitrectomy, prevention

\section{INTRODUCTION}

Eye injuries are one of the main reasons for severe visual impairment. Eighteen million people worldwide have uniocular blindness from traumatic injury.According to Kuhn, eye injuries could be classified as closed globe or open globe injuries. Closed-globe injuries are more common than open globe; however, the latter have poorer visual prognosis [1"publisher":"Thieme","number-ofpages":"508","source":"Google Books","abstract":"Ocular Trauma: Principles and Practice is the first comprehensive clinical reference on ocular injuries in more than a decade. Each chapter gives you detailed instructions on evaluation, treatment, and management, including what to do, how to do it, and why to do it. You'll also learn techniques for developing individualized treatment strategies for hard-toidentify injuries. The book begins with general terminology and classification of ocular trauma, and goes on to cover such topics as: the new role of endoscopy; eye restoration with complete iris loss; anterior chamber disorders; rehabilitation advances; medicolegal issues regarding the ophthalmologist as an expert witness; and much more! Insightful chapters written by patients offer their perspectives on the physical injuries and emotional trauma they have experienced.This reader-friendly book is a valuable addition to the professional library of every ophthalmologist, retina specialist, resident, and emergency room physician who deals with trauma patients. No other resource gives you such an abundance of information on assessment, emergency intervention, management, and rehabilitation. Make sure you have the best, state-of-the-art information as you treat patients who

Address for correspondence: Jerzy Mackiewicz, Department of Vitreoretinal Surgery, Medical University of Lublin

E-mail:jerzymackiewicz@umlub.pl

Received:26 May 2016; accepted: 12 July 2016; first published on February 2017 have suffered an ocular injury. Key features of OCULAR TRAUMA: A systemic approach to eye management on a tissue by tissue basis, instead of the traditional anterior/ posterior approach Practical, easy-to-use format, featuring more than 350 illustrations (156 in full color].

Most injuries follow domestic accidents and predominantly affect children (21.4-47.3\%). Males are more exposed to ocular trauma than females. The most frequent causes of eye injuries in men are related to outdoor activities and work [2, 3800 annual lawnmower-related injuries are treated in the USA, with 9,300 involving children. This work reviews the literature and reports our 10-year experience with lawnmower injuries. We retrospectively reviewed patients with lawnmower injuries admitted to the Women and Children's Hospital of Buffalo from 1995 to 2005 and reviewed the literature. Sixteen patients with mower-related injuries were admitted to our institution, with three due to walk-behind mowers and 13 due to riding mowers. The mean age was 7.5 years. Riding mower injuries had a higher average injury severity score (14.3 vs. 9].

Lawn mowers have been shown to cause serious injuries and the number of such accidents has been increasing over time. The high speed of the out-thrown objects makes them potentially destructive. Injuries from projectiles, typically rocks, wires or glass, account for $17 \%$ of lawn mower injuries[2, 3800 annual lawnmower-related injuries are treated in the USA, with 9,300 involving children. This work reviews the literature and reports our 10-year experience with lawnmower injuries. We retrospectively reviewed patients with lawnmower injuries admitted to the Women and Children's Hospital of Buffalo from 1995 to 2005 and reviewed the literature. Sixteen patients with mower-related injuries were admitted to our institution, with three due to walk-behind mowers and 13 due to riding mowers. The mean age was 7.5 years. Riding mower injuries 
had a higher average injury severity score (14.3 vs. 9]. The major group of victims are children, mostly boys, of mean age 10.7 years. The most common injuries described were: laceration (41.2\%), soft tissue injury (21.4\%), burn (?) buttock/ coccyx (15.5\%), and bone fracture (10.3\%)[4or 11.1 injuries per 100000 US children per year. The mean age was 10.7 (SD: 6.0]. An epidemiological survey conducted in the USA showed that lawn mowers were responsible for about 81,907 injuries between 2000 - 2009. It appeared that men, people aged 40-49, and Caucasians were the groups most likely to be injured, with the head, specifically the eye, as most commonly injured part of the body (42.5\%) [5and some of the more frequency used equipment (e.g., lawn mowers].

The range of eye damage inflicted vary, from superficial skin damage and corneal epitheliumerosions, through intraocular haemorrhages caused by blunt injury, to penetrating injuries damaging all the eye structures. Some of the injuries may be treated with just topical medication, more serious ones may require surgical intervention. Some potentially blindening injuries may remain asymptomatic for a considerable period of time, due to the small size of projectile and its high velocity which makes it able to penetrate corneal tissue, but stop in i.e lens.

This study presents 4 case reports of lawn mower-related injuries treated at the Department of Vitreoretinal Surgery of the Clinical Hospital in Lublin between 2013-2015.

\section{MATERIALS AND METHOD}

A retrospective study of 4 patients admitted to the Department of Vitreoretinal Surgery in Lublin between 2013 - 2015 after severe open or closed globe injury, one with an intraocular foreign body (IOFB), during lawn mower use.

The patients with open globe injury required surgical treatment due to corneoscleral lacerations, damage of the lens and iris, and IOFB in the vitreous cavity. Primarily, the wounds were sutured. Three patients then underwent pars plana vitrectomy, one with IOFB removal from the vitreous cavity.The patient with closed globe injury required topical treatment only.

\section{CASE 1}

A 46-year-old male suffered a penetrating injury to his left eye with a projectile whilst using an electric rotary lawn mower in his yard, without any kind of eye protection. Corneoscleral lacerations were primarilyy repaired in the regional hospital. Two weeks later he was admitted to our department with visual acuity of light perception with proper light projection. A wide, about $4 \mathrm{~mm}$ in length, corneoscleral wound was properly sutured, hyphaema, iridodonesis and lens opacity were observed. Further eye segments were not visible in the slit lamp due to the hyphaema. Ultrasound examination showed a foreign body entrapped in the posterior segment, $3 \times 2 \mathrm{~mm}$ in size, with associating vitreous hemorrhage. In radiological examination, a foreign body of similar in size was reported. The patient underwent an immediate vitrectomy with constant antibiotic flow during which the intraocular foreign body was removed from the posterior pole of the eye. Remnants of the lens present in the vitreous cavity were aspirated with the vitrectome. A laser was placed over the optic disc, where the IOFB was stuck, followed by C3F8 gas injection. The eye was left aphakic. Two days later, the patient was discharged from the Department with visual acuity of hand movement and proper light projection.

Subsequently, 2 months later, he developed retinal detachment, which was treated the next day by vitrectomy with retinotomy and silicone oil tamponade. Full retinal apposition was achieved.

Two months later, his visual acuity was light perception with improper projection. Intraocular pressure was normal during the follow-up. At the last examination he presented corneoscleral sutures in place, a deep and clear anterior chamber and aphakia. In the posterior segment - the vitreous cavity filled with silicone oil, retinal scars in place of laser therapy, and scarring in the retinoscleral wound after the foreign body removal. Retina was attached.

Eventually, the patient, aware of the trauma severity, was content with the surgery outcome.

\section{CASE 2}

A 63-year-old male was admitted to our hospital as an emergency with a penetrating injury of the right eye, sustained while using a hand lawnmower without any eye protection. On examination, the visual acuity was reduced to hand movement, with improper light projection. The eye was hypotonic, with a corneoscleral wound and iris prolapse. In the anterior chamber, multiple glass splinters, hyphaema and viterous were present. In ultrasound examination, choroid elevation was reported. No intraocular foreign bodies of the posterior segment were found in USG, nor in CT scans.

As the first step of treatment, the corneoscleral wound was sutured, splinters removed, and the iris put in place. Anterior vitrectomy was performed to remove the vitreous from the anterior chamber, with $0.1 \mathrm{ml}$ cefuroxyme injection at the end of the procedure. When the choroidal elevation receded, lens removal was performed by means of the phacoemulsification technique, synechiolysis with iris plastics, and the lens was then inserted into the posterior capsule.

On the last examination, 2 months after surgery, visual acuity was 0.4 on the Snellen scale, sutures were in place, clear anterior chamber was present with good outcome of iridoplastics, and correct lens position. Patient was very satisfied with the therapeutical outcome.

\section{CASE 3}

A 63-year-old woman presented to the clinic reporting eye trauma while using a lawn mower, without eye protection. Visual acuity of light perception with improper projection, intraocular pressure of $27 \mathrm{mmHg}$ was measured. Physical examination showed a large corneal epithelium defect, with no perforating wounds. Hyphaema of approximately $5 \mathrm{~mm}$, and scattered blood in the rest of the anterior chamber was observed. USG examination did not reveal IOFB presence, vitreous hemorrhage or retinal detachment. After 2 days of topical treatment, including dorzolamide/timolol, fluorochinolone antibiotics eyedrops, and artificial tears, she was discharged from the department with visual acuity of 0.7 , and IOP of $10 \mathrm{mmHg}$. On follow-up examination, her visual acuity remained at the same level. No further ophthalmic complications were observed.

\section{CASE 4}

A 63-year-old man presented to the hospital after suffering a blunt trauma with a projectile from underneath a lawn mower. He reported lack of any eye protection. The eye that 
suffered injury was the only functional one, with the second of visual acuity of fingers count of $0.5 \mathrm{~m}$, due to meningioma located in the area of nerves crossing, and following surgery. On examination, he presented with vision acuity of fingers count to $0.5 \mathrm{~m}$, and IOP raised to $40 \mathrm{mmHg}$. In slit lamp examination, corneal epithelium defect, hyphaema in anterior chamber, and subluxated lens with wide, non-responsive pupil were present. USG examination in B projection showed neither retinal detachment nor vitreous haemorrage. The patient was treated with acetazolamide orally, and topical dorzolamid combined with brymonidine. After regaining normal IOP, the patient was discharged from hospital. After 6 months he was treated with vitrectomy combined with lensectomy procedure, and subsequently, IOL was positioned with iris plastics. The patients was finally discharged with visual acuity of 0.2 , and IOP of $18 \mathrm{mmH}$ during follow-up.

\section{SUMMARY}

To conclude, an improvement in local condition was achieved in 3 of 4 cases, and stabilization in one case. An intraocular foreign body was removed, wounds sutured and damaged tissues placed in position. All eyeballs were saved. The visual outcome for each patient is presented in Table 1. It can be noticed that in one patient who did not achieve improvement in vision, proper intervention did not take place until 2 weeks after the trauma, which probably strongly affected the final outcome. All patients treated properly in a short period of time after injury presented a significant improvement in visual acuity during the follow-up.

All patients suffered eye injury during domestic activities. None of them had used ocular protection during lawn mowing as they were unaware of its potential risk.

Table 1. Patients' visual outcome on admission and at follow-up examination

\begin{tabular}{cll}
\hline Patient No. & Visual Acuity on admission & Final Visual Acuity \\
\hline 1 & CF, proper projection & CF, improper projection \\
\hline 2 & HM, improper projection & 0.4 , proper projection \\
\hline 3 & CF, improper projection & 0.7, proper projection \\
\hline 4 & CF, proper projection & 0.2, proper projection \\
\hline
\end{tabular}

HM- Hand Movement; CF- Count Fingers

\section{DISCUSSION}

The four cases presented show the potential damage that can be inflicted while using a lawn mower. There is no existing data on the number of lawn mowers in Poland, but it can be estimated that they are present in the majority of households. The presented study did not include the more common, less severe cases that are more likely to end with superficial damage[2], but focused on those where an intraocular foreign body or severe trauma with significant visual decline was present. The presence of an intraocular foreign body is a common and serious problem accounting for approximately $17 \%$ to $41 \%$ of penetrating ocular injury [6, 7London. Patients who underwent a primary surgical repair and removal of a posterior segment IOFB were identified using a hospital database. Of the 140 patients identified, 114 (81\%]. It has been shown that ocular trauma from posterior segment IOFB remains a major cause of visual loss in young adults [6], and should be approached aggressively to reduce the occurrence of postoperative retinal detachment, and thereby improve visual outcome [8]. In the current study, three of four patients were qualified for pars plana vitrectomy procedure, either as a primary treatment, or to handle the posttraumatic complications. Vitrectomy is a treatment of choice in cases with IOFB, retinal detachment, persistent vitreous haemorrhage or endophthalmitis. The outcome varies, depending on the localization and severity of trauma. Patients presenting with open globe injuries and retained IOFBs have a tendency to develop peripheral vitreous traction and retinal tears which ultimately lead to retinal detachment [8]. Even with the success of the primary vitrectomy, numerous potential complications were reported: retinal detachment, proliferative vitreoretinopathy, strabismus, epiretinal membrane, glaucoma and endophtalmitis [6-9London. Patients who underwent a primary surgical repair and removal of a posterior segment IOFB were identified using a hospital database. Of the 140 patients identified, 114 (81\%]. Overall, 5-31\% patients have a final visual acuity of less than $3 / 60$, with an increasing number of more successful operations corresponding with the advances in vitreoretinal surgery $[6,7,9$ London. Patients who underwent a primary surgical repair and removal of a posterior segment IOFB were identified using a hospital database. Of the 140 patients identified, 114 (81\%].

Most eye injuries to the elderly occur at home and in men. The risk of ocular injury from the use of powered lawn mowers has not been widely enough documented in the medical literature. Even though most the cases have proved to be minor corneal erosions, lawn mowers have been shown to cause serious injuries and these incidents have been increasing over time. The high speed of the outthrown objects makes even small stones highly destructive. With rotary blades reaching 3,000 rpm, an $0.5 \mathrm{~kg}$ object can be launched with speed of over $300 \mathrm{~km} / \mathrm{h}$ [2, 3800 annual lawnmowerrelated injuries are treated in the USA, with 9,300 involving children. This work reviews the literature and reports our 10year experience with lawnmower injuries. We retrospectively reviewed patients with lawnmower injuries admitted to the Women and Children's Hospital of Buffalo from 1995 to 2005 and reviewed the literature. Sixteen patients with mowerrelated injuries were admitted to our institution, with three due to walk-behind mowers and 13 due to riding mowers. The mean age was 7.5 years. Riding mower injuries had a higher average injury severity score (14.3 vs. 9], which is why wearing protective eyewear is essential and should be advised to anyone using lawn mowers, or lawn trimmers [5and some of the more frequency used equipment (e.g., lawn mowers]. Other types of passive protection, like grass catchers, deflectors and guards should also be fitted to modern lawn mowers in an effort to reduce the number of missile injuries. This should be followed with the easily seen placement of those devices to people buying lawn mowers, and proper information about their safe use. As Whittaker et al. show in a survey taken among over 200 patients using lawn mowers, only $4 \%$ of them used such eye protection. In most cases, lack of knowledge about potential harm was the reason of not using protection [10]. Furthermore, the packaging of only 1 of 25 models of lawn mower examined recommended the use of ocular protection [4or 11.1 injuries per 100000 US children per year. The mean age was 10.7 (SD: 6.0] 
In a large retrospective study presenting lawn mower trauma to children in 14 consecutive years, basic guidelines for manufacturers were proposed. They included more rigorous performance provisions regarding prevention of penetration of feet and toes under the mower and into the path of the blades, shielding of hot mower parts from access by young children, and equipping all ride-on lawn mowers with a no-mow-in-reverse default feature, with the location of its override switch behind the seating position of the rideon mower operator. This would require the operator to look behind the mower before mowing in reverse [4or 11.1 injuries per 100000 US children per year. The mean age was 10.7 (SD: 6.0]. Recognizing the problem, the American Academy of Ophthalmology (AAO) has already introduced suggestions for safe mowing (Tab. 2), but there is no official statement from the Polish Society of Ophthalmology (PTO) about the potential risks associated with unprotected lawn-mowing.

Table 2. Recommendations of American Academy of Ophthalmology related to safe lawn mowering

\begin{tabular}{l} 
Remove sticks, glass, metal, wire, and/or stones from the area you are working; \\
Check your mower and tractor blades. If the tips are thin or worn, they can break \\
off and become dangerous missiles \\
\hline Watch for trees with low-hanging branches \\
\hline Keep bystanders out of the 'line of fire' - at least 10 yards away \\
\hline Use a grasscatcher \\
Mow Orly Turing daylight \\
Wear eye protection while mowing. Regular eyeglasses or contact lenses will \\
not protect you from eye hazards. You must wear protective eyewear over your \\
corrective lenses. Polycarbonate safety goggles that meet ANSI Z87 standards are \\
ideal
\end{tabular}

\section{CONCLUSIONS}

Lawn mower eye injuries are a significant cause of serious visual acuity impairment or blindness. The tragedy is keenly felt in the realization that these devastating injuries could all be prevented. Strategic preventative measures should be developed through partnership between the medical profession, the media, industry specialists and the wider community. Passive protection that is provided by safer product design is the strategy with the highest likelihood of success in preventing these ongoing injuries. Rural areas should be considered as those in need of special education, where various studies have revealed very low protection usage $[11,12]$. With children being very susceptible to this kind of injury, it is of most importance to educate parents not to allow them to use lawn mowers on their own, and to take extra caution with children being present during lawn mower use. The presented study has shown that lawn mower eye injuries are still a therapeutic, social and economic problem.

\section{REFERENCES}

1. Kuhn F, Pieramici DJ. Ocular Trauma: Principles and Practice. Thieme; 2011.

2. Mullins J. Lawn mower injuries: a review. J Emerg Nurs JEN Off Publ Emerg Dep Nurses Assoc. 2010;36(1):83-84. doi:10.1016/j. jen.2009.10.008.

3. Lau ST, Lee Y-H, Hess DJ, Brisseau GF, Keleher GE, Caty MG. Lawnmower injuries in children: a 10-year experience. Pediatr Surg Int. 2006;22(3):209-214. doi:10.1007/s00383-005-1633-0.

4. Vollman D, Smith GA. Epidemiology of lawn-mower-related injuries to children in the United States, 1990-2004. Pediatrics. 2006;118(2):e273-e278. doi:10.1542/peds.2006-0056.

5. Leinert J, Griffin R, Blackburn J, McGwin G. The epidemiology of lawn trimmer injuries in the United States: 2000-2009. J Safety Res. 2012;43(2):137-139. doi:10.1016/j.jsr.2012.03.001.

6. Wickham L, Xing W, Bunce C, Sullivan P. Outcomes of surgery for posterior segment intraocular foreign bodies--a retrospective review of 17 years of clinical experience. Graefes Arch Clin Exp Ophthalmol Albrecht Von Graefes Arch Für Klin Exp Ophthalmol. 2006;244(12):1620-1626. doi:10.1007/s00417-006-0359-6.

7. Chiquet C, Zech JC, Gain P, Adeleine P, Trepsat C. Visual outcome and prognostic factors after magnetic extraction of posterior segment foreign bodies in 40 cases. Br J Ophthalmol. 1998;82(7):801-806.

8. Erakgun T, Egrilmez S. Prognostic factors in vitrectomy for posterior segment intraocular foreign bodies. J Trauma. 2008;64(4):1034-1037. doi:10.1097/TA.0b013e318047dff4.

9. Greven CM, Engelbrecht NE, Slusher MM, Nagy SS. Intraocular foreign bodies: management, prognostic factors, and visual outcomes. Ophthalmology. 2000;107(3):608-612.

10. Whittaker KW, Baldwin HC, Ikram K, Luff AJ. Attitudes towards protective eye-wear: ocular trauma from lawn mowers. Injury. 1999;30(2):144-145.

11. Casson RJ, Walker JC, Newland HS. Four-year review of open eye injuries at the Royal Adelaide Hospital. Clin Experiment Ophthalmol. 2002;30(1):15-18. doi:10.1046/j.1442-9071.2002.00484.x.

12. Northey LC, Bhardwaj G, Curran S, McGirr J. Eye trauma epidemiology in regional Australia. Ophthalmic Epidemiol. 2014;21(4):237-246. doi: 10.3109/09286586.2014.928825. 